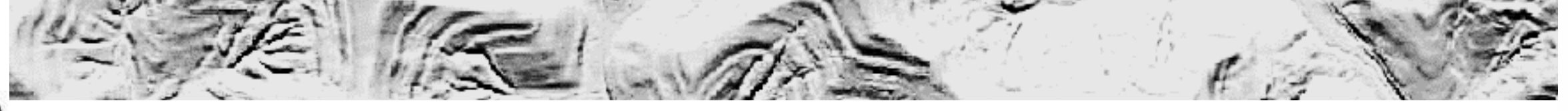

\title{
Late Cenozoic Drainage Reorganization of Former St. Lawrence Drainage Left a Legacy of On-Going Disequilibria in Upper Ohio River Basin Fluvial and Colluvial Systems
}

\author{
J. Steven Kite ${ }^{1}$ (ikite@wvu.edu) \\ Mark D. Swift 1, 2
}

1 West Virginia University Department of Geology \& Geography ${ }^{2}$ Washington \& Jefferson College Department of Music

Session T71: The New Appalachians: Cenozoic Deformation, Drainage Reorganization, and Landscape Disequilibrium in a Paleozoic Orogen

Paper 63-4 https://gsa.confex.com/gsa/2019AM/meetingapp.cgi/Paper/339747

2:20-2:40 PM, Sunday, 22 September 2019

229A North Building, Phoenix Convention Center

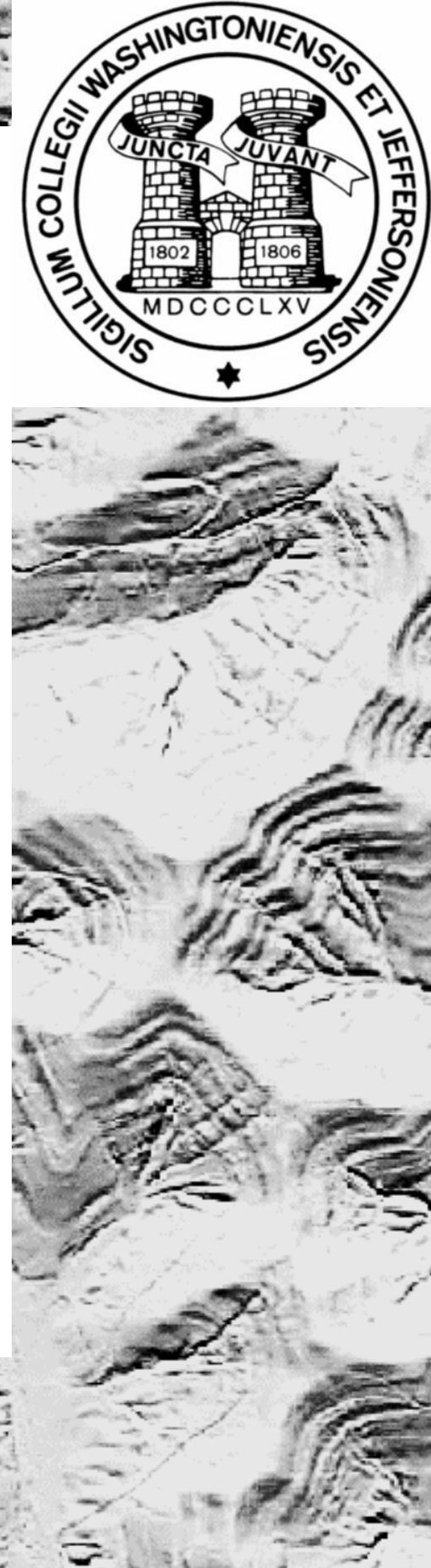




\section{Generalized Reconstruction} stream flove

Labrador Sea Miocene North America Drainage Mackenzie
Mountains

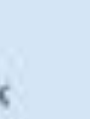
Paleo-Bed
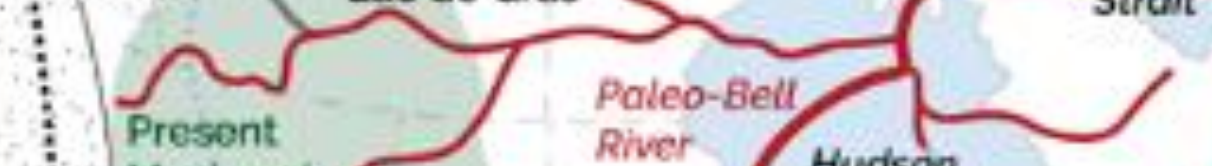

Mackenzie

River Basin

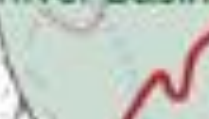

cypress Hill

용

Pacific

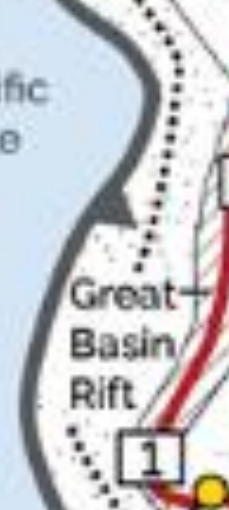

3)
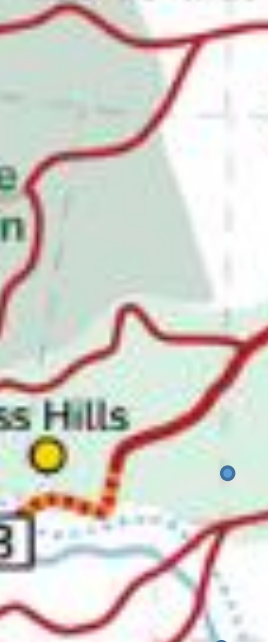

River
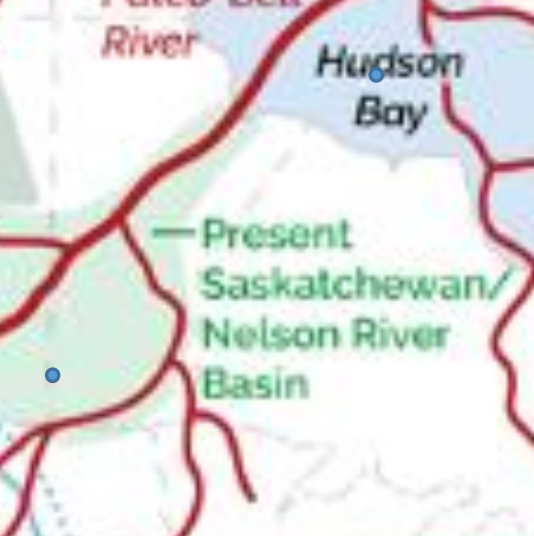

.

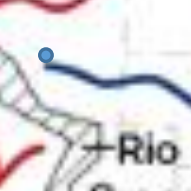

Polco-Saint

Loiwrence River
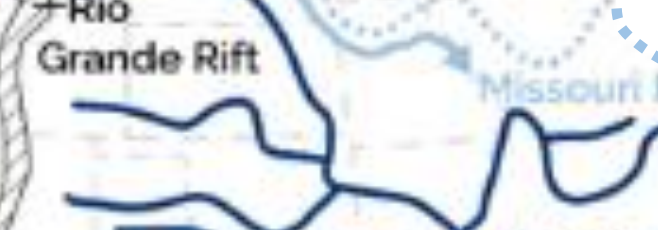

Paleo-Mississipp

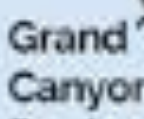

Region

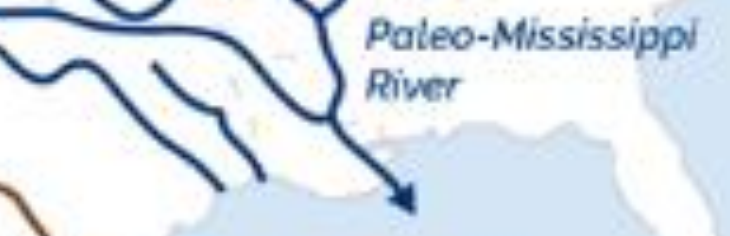

Labrador

\section{Key Points}

$\rightarrow$ St. Lawrence Drainage Rearrangement Caused

Possible Maximum Southeast Extent of St. Lawrence Basin Incision \& Knickzones

$\rightarrow$ Incision Led to Regional Slope Instability

$\rightarrow$ Both Are On-Going 


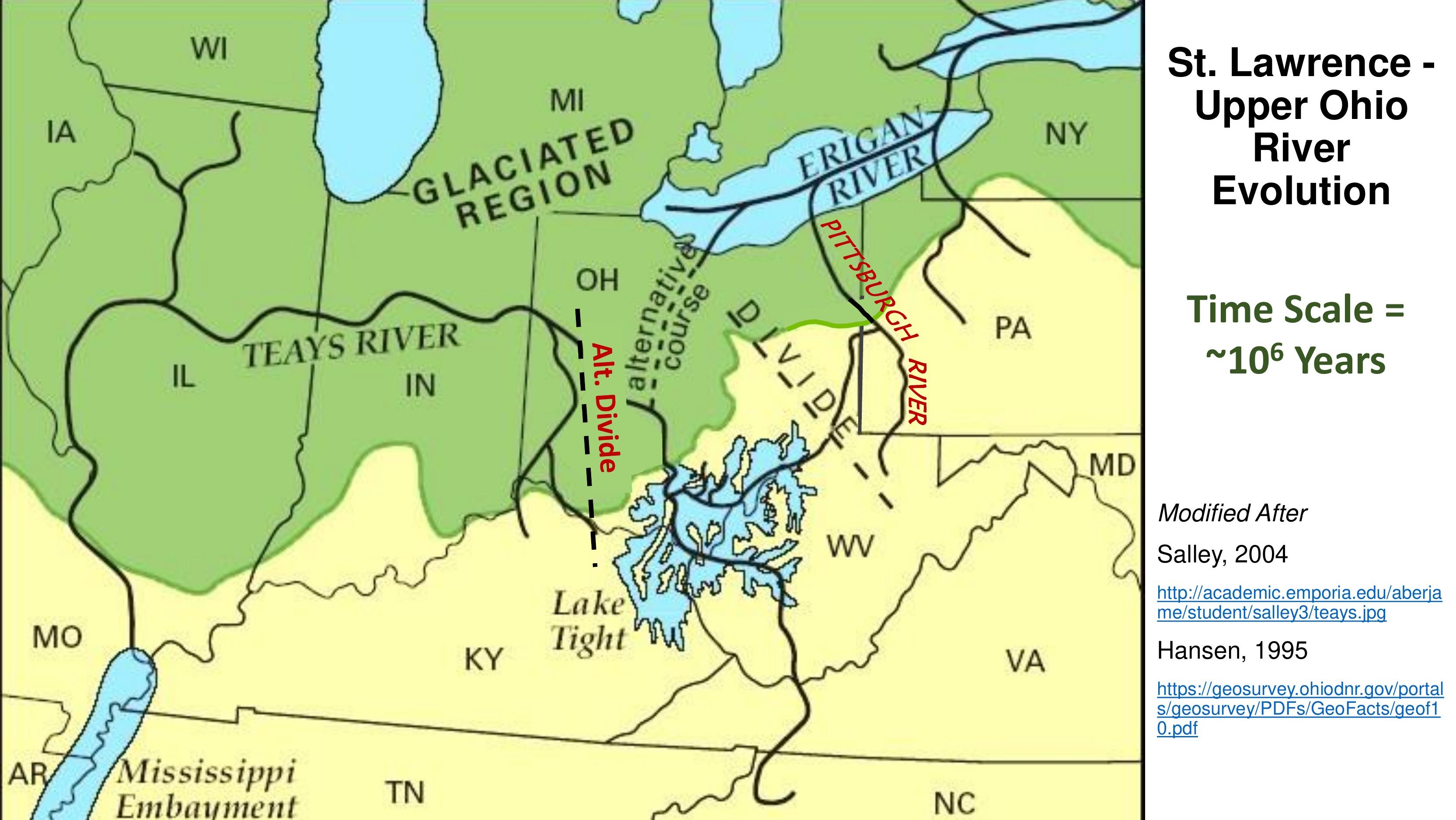


Teays - Ohio

River Systems

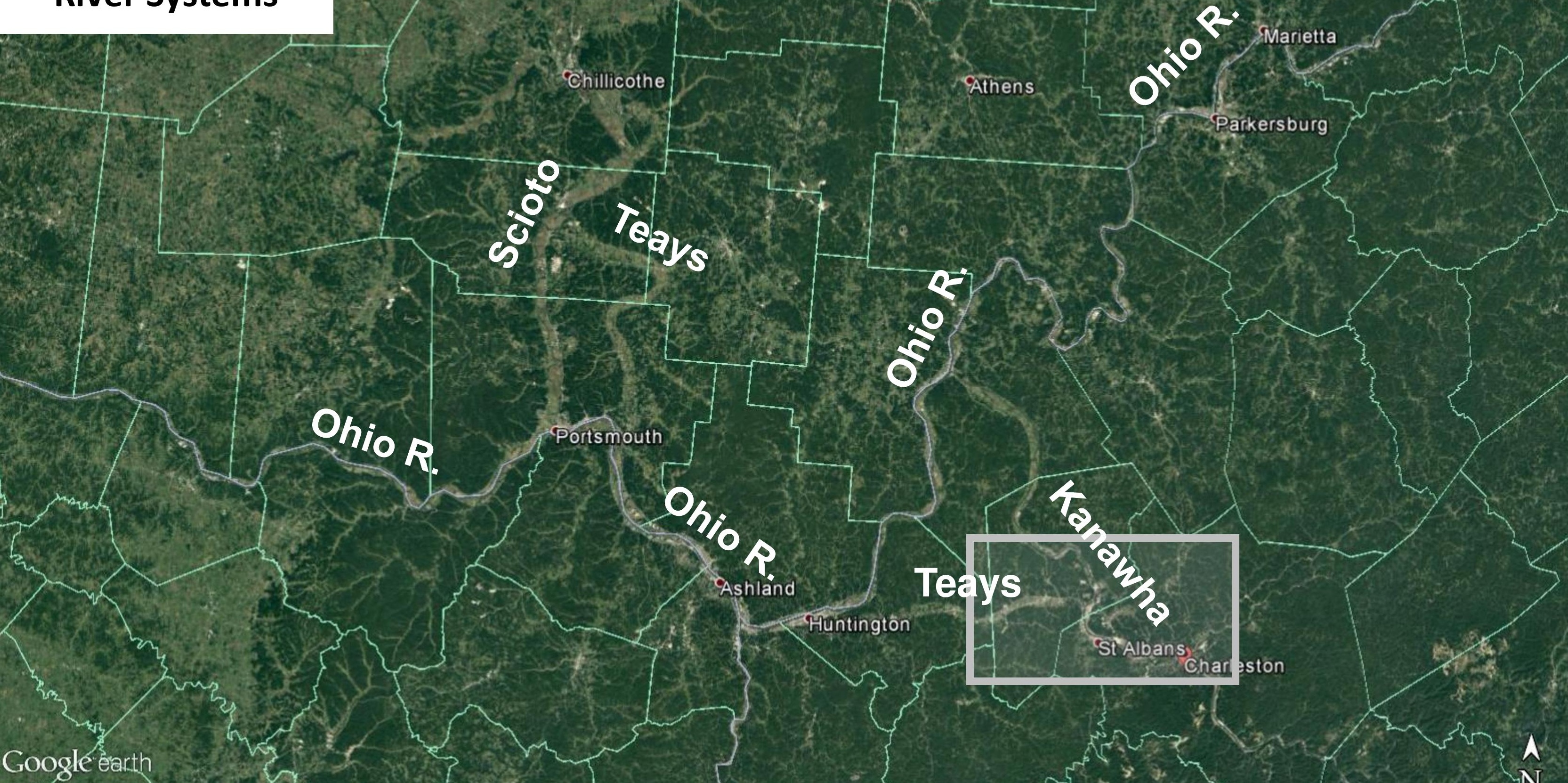




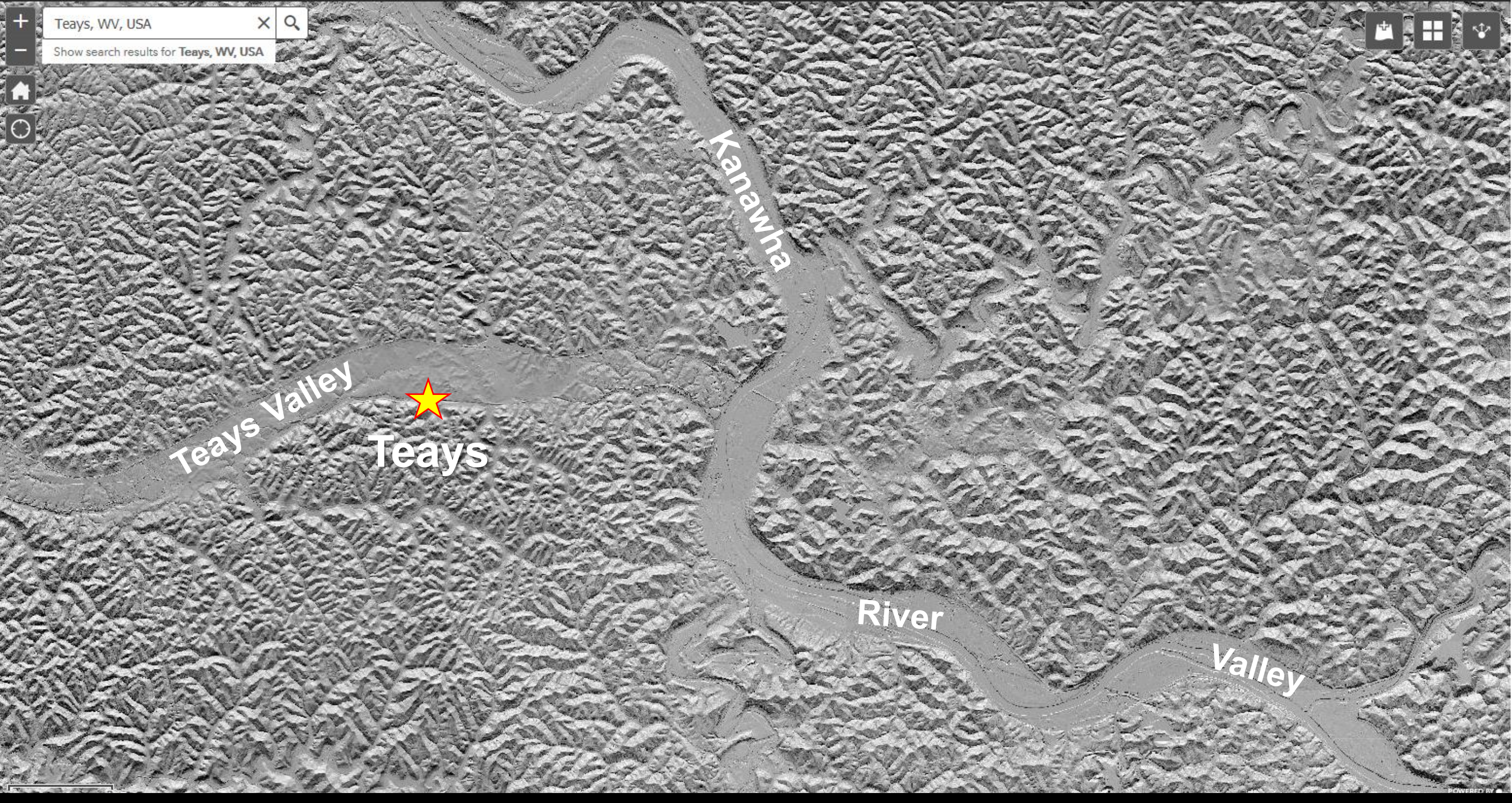

\section{Teays Valley \& Kanawha (Lower New) River Valley, West Virginia}




\section{Generalized Reconstruction Miocene North America Drainage}

Labrador Sea

Mackenzie stream flove

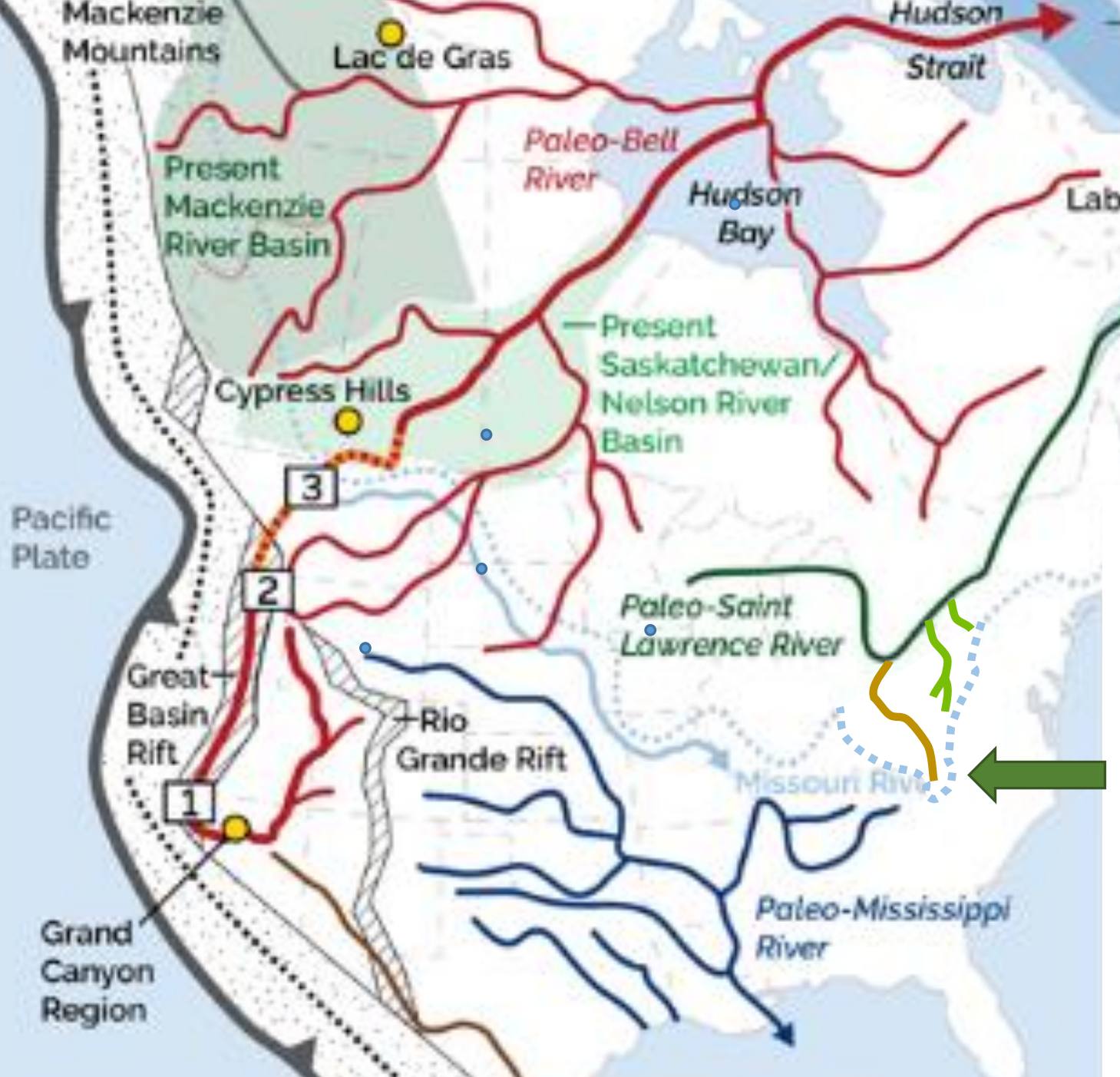

Modified from Cantner \& Jackson, 2018 (https://www.earthmagazine.org/article/paleo-bellriver-north-americas-vanished-amazon), which is based on Sears, 2013, GSA Today

\section{Basin Configuration}

Existing St. Lawrence

Existing St. Lawrence + Pittsburgh Paleo-River

Existing St. Lawrence + Teays \& Pittsburgh Paleo-Rivers
Area $\mathbf{k m}^{2} \quad$ Diff.

$1,344,200$

$1,411,200+4.8 \%$

$1,525,200$ $+11.9 \%$ 

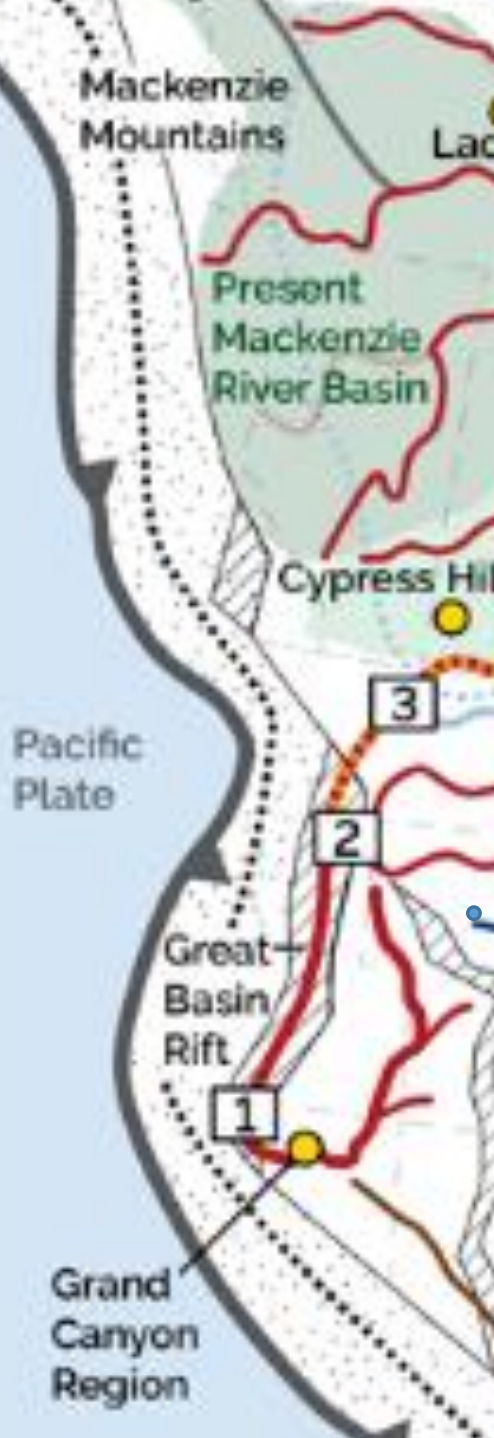

de Gras

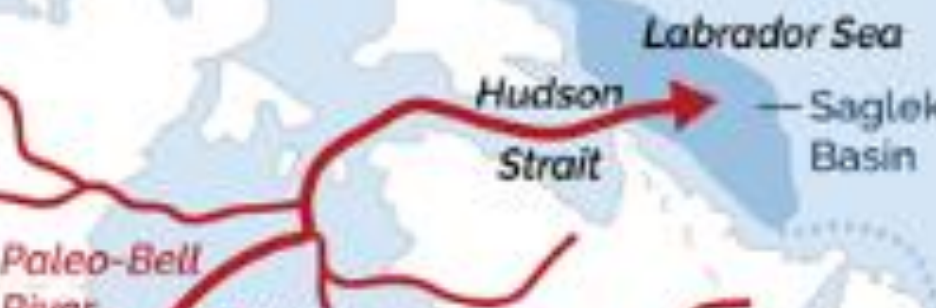

River

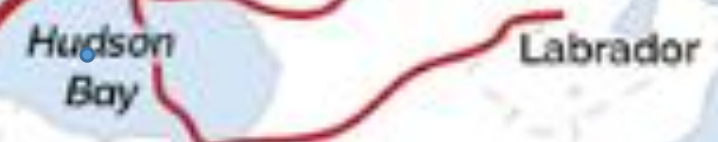

Labrador

\section{$\Omega \quad-$ Present}

Saskatchewa

Nelson River Basin

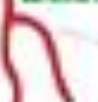

Poleo-Saint Loiwrence River
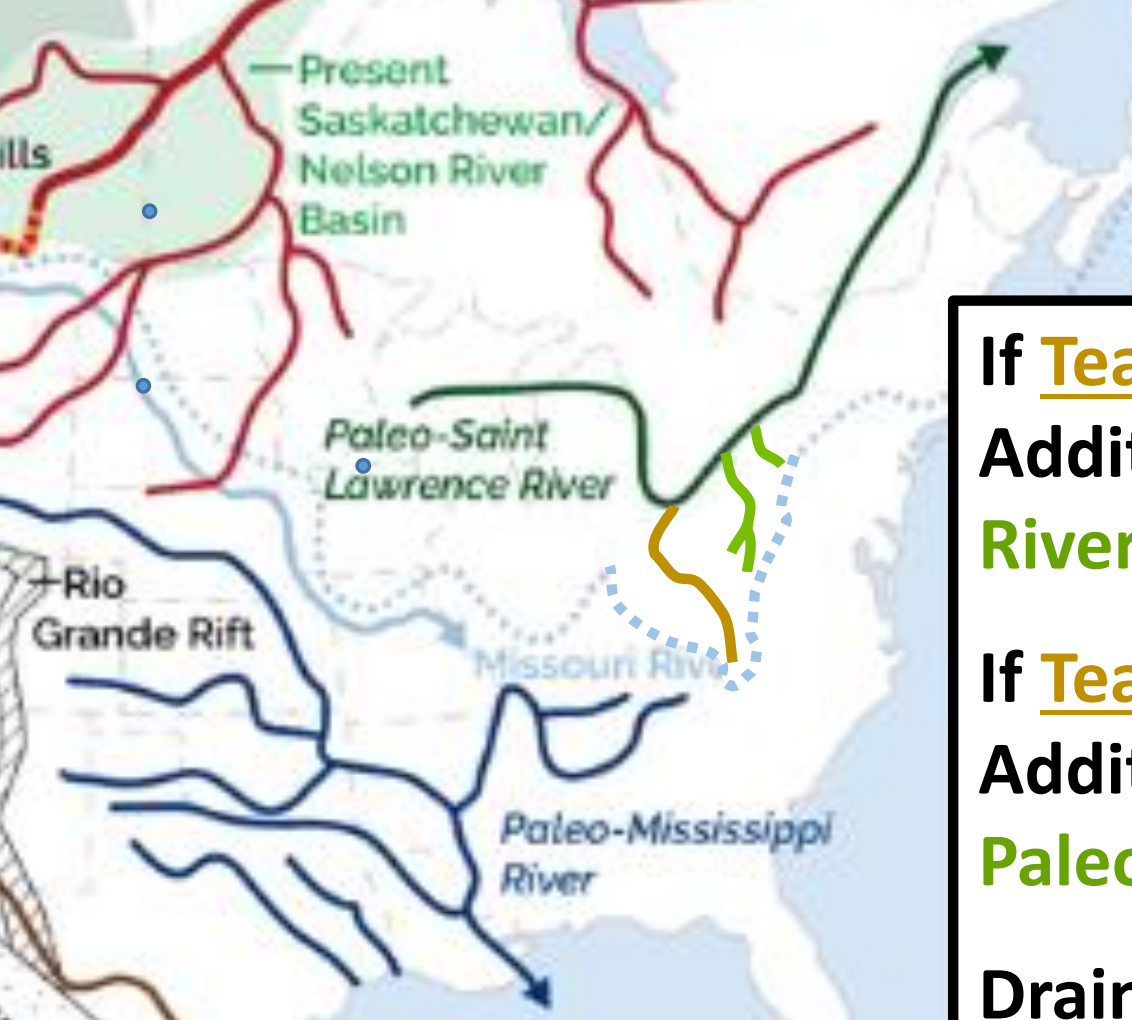

If Teays River Was Not Part of St. Lawrence River Basin, Addition of Pittsburgh \& Ancestral Allegheny PaleoRivers Increased Ohio River Drainage Area by $12 \%$

If Teays River Was Part of St. Lawrence River Basin, Addition of Teays, Pittsburgh, \& Ancestral Allegheny Paleo-Rivers Increased Ohio River Basin Area by $\mathbf{3 4} \%$ Drainage Area for Headwater Creeks at Breached Cols Increased by $>10,000 \%$ 


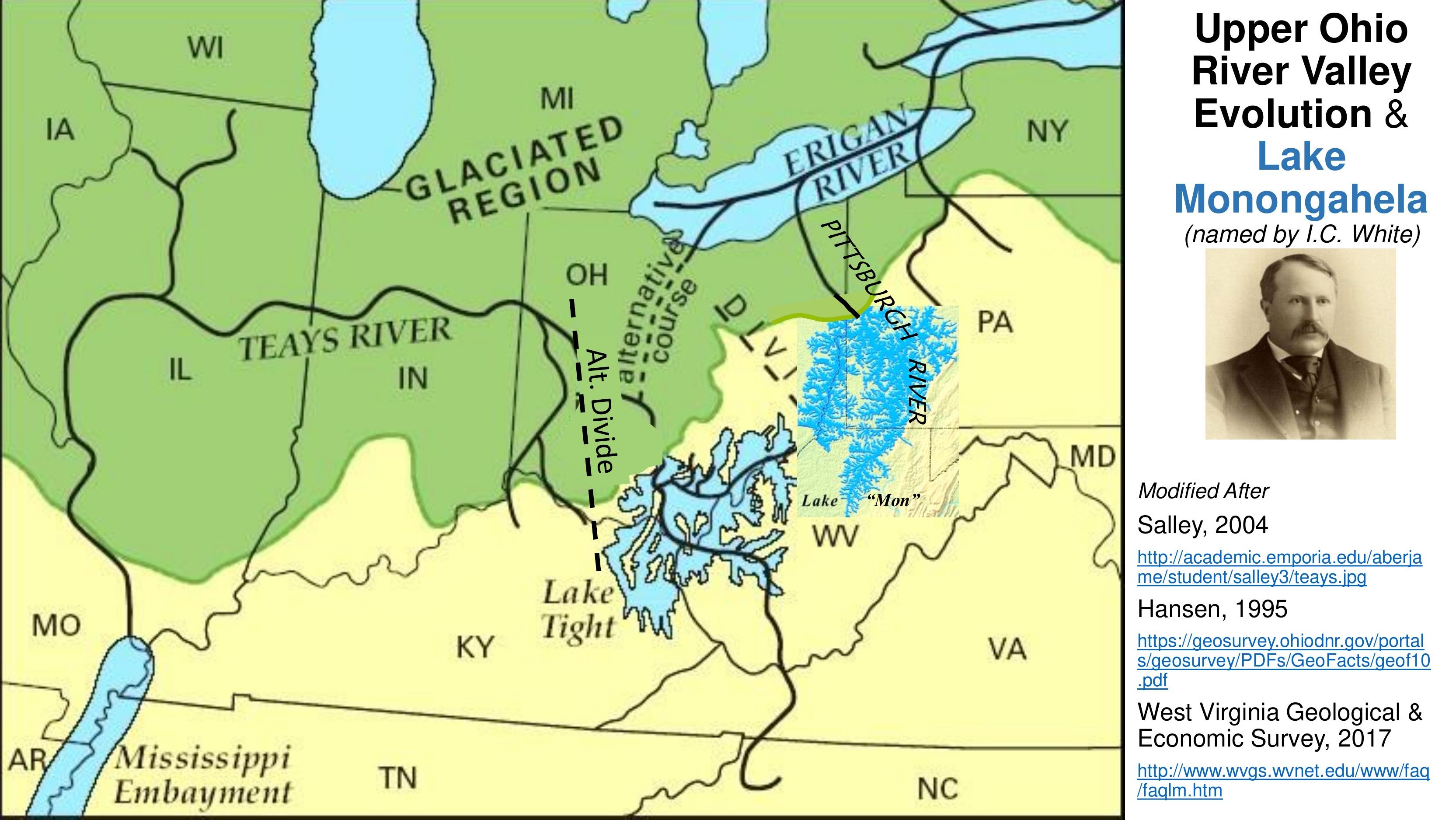


Lake Sediment Samples

Typical Carmichaels Laminated Reddish Silt-Clay

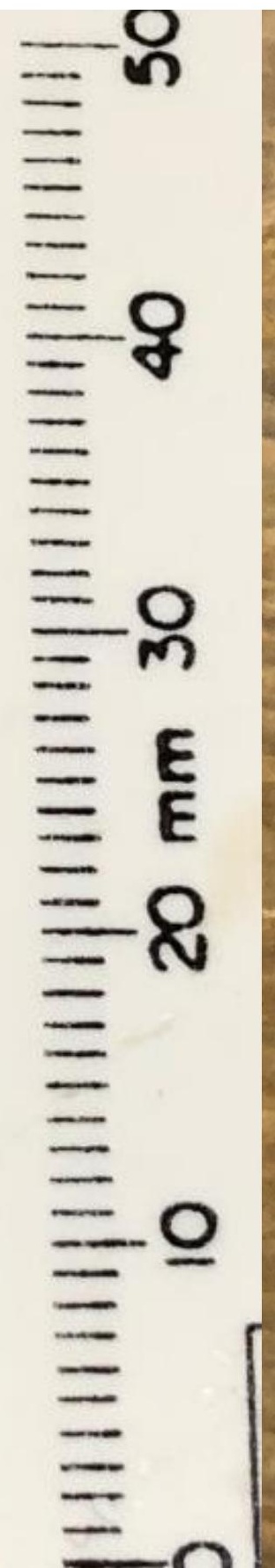

Carmichaels "Pottery Clay"

Finely Laminated White Clay

$-0$

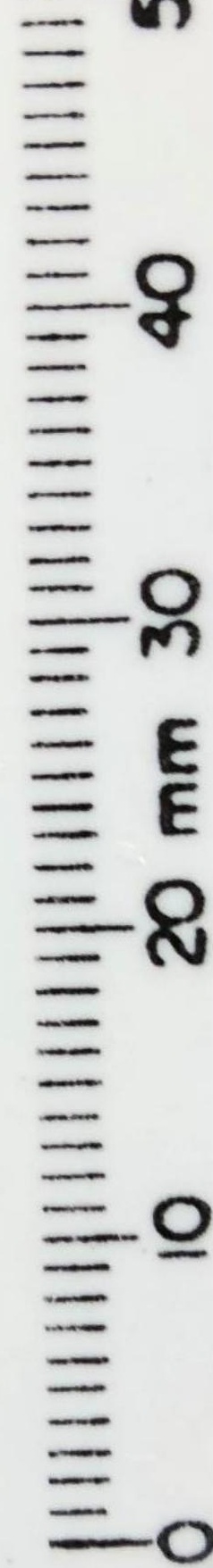


Lake Monongahela Age Assignments

\begin{tabular}{|c|c|}
\hline Source & Age \\
\hline White 1896 & Pre-Illinoian \\
\hline Campbell 1902 & Pre-Illinoian (Kansan or Pre-Kansan) \\
\hline Leverett 1934 & Pre-Illinoian, Illinoian \& Wisconsin \\
\hline Lessig 1963 & Pre-Illinoian; $1^{\text {st }}$ regional glaciation \\
\hline $\begin{array}{l}\text { Clendening, et al. } 1967 \\
\text { Gillespie \& Clendening } 1968\end{array}$ & $\begin{array}{l}\text { Farmdalian (Wisconsin) } \\
\begin{array}{ll}22,000 \pm 100014 \mathrm{C}-\mathrm{BP} & {[26,394 \pm 1262 \text { CalBP }]} \\
>33,00014 \mathrm{C}-\mathrm{BP} & {[>37,500 \mathrm{CalBP}]}\end{array}\end{array}$ \\
\hline $\begin{array}{l}\text { Jacobson 1985, } 1987 \\
\text { Jacobson et al. } 1988\end{array}$ & $\begin{array}{l}\text { Pre-Illinoian, Illinoian, (Wisconsin Low Terraces) } \\
\text { > 40,000 14C-BP [> 43,700 CalBP] } \\
<780,000 \text { BP (Brunhes) } \\
>780,000 \text { BP (Matuyama) }\end{array}$ \\
\hline $\begin{array}{l}\text { Behling \& Kite } 1988 \\
\text { Kite unpublished data } \\
\text { Morgan } 1994\end{array}$ & 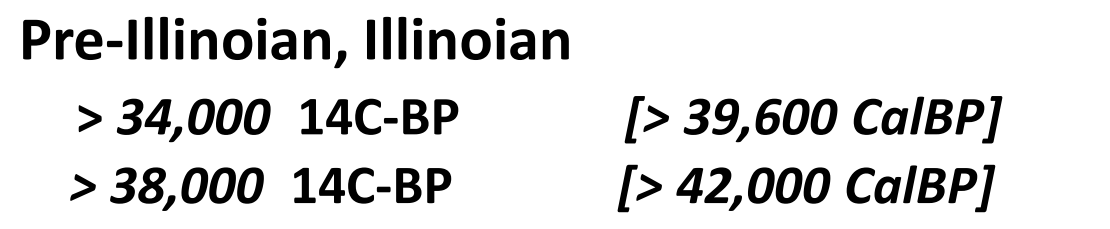 \\
\hline $\begin{array}{l}\text { Marine 1997; Marine \& } \\
\text { Donahue } 2000\end{array}$ & $\begin{array}{l}\text { Pre-Illinoian \& Illinoian } \\
<780,000 \text { BP (Brunhes) }\end{array}$ \\
\hline
\end{tabular}

Illinois State Geological Survey 2018

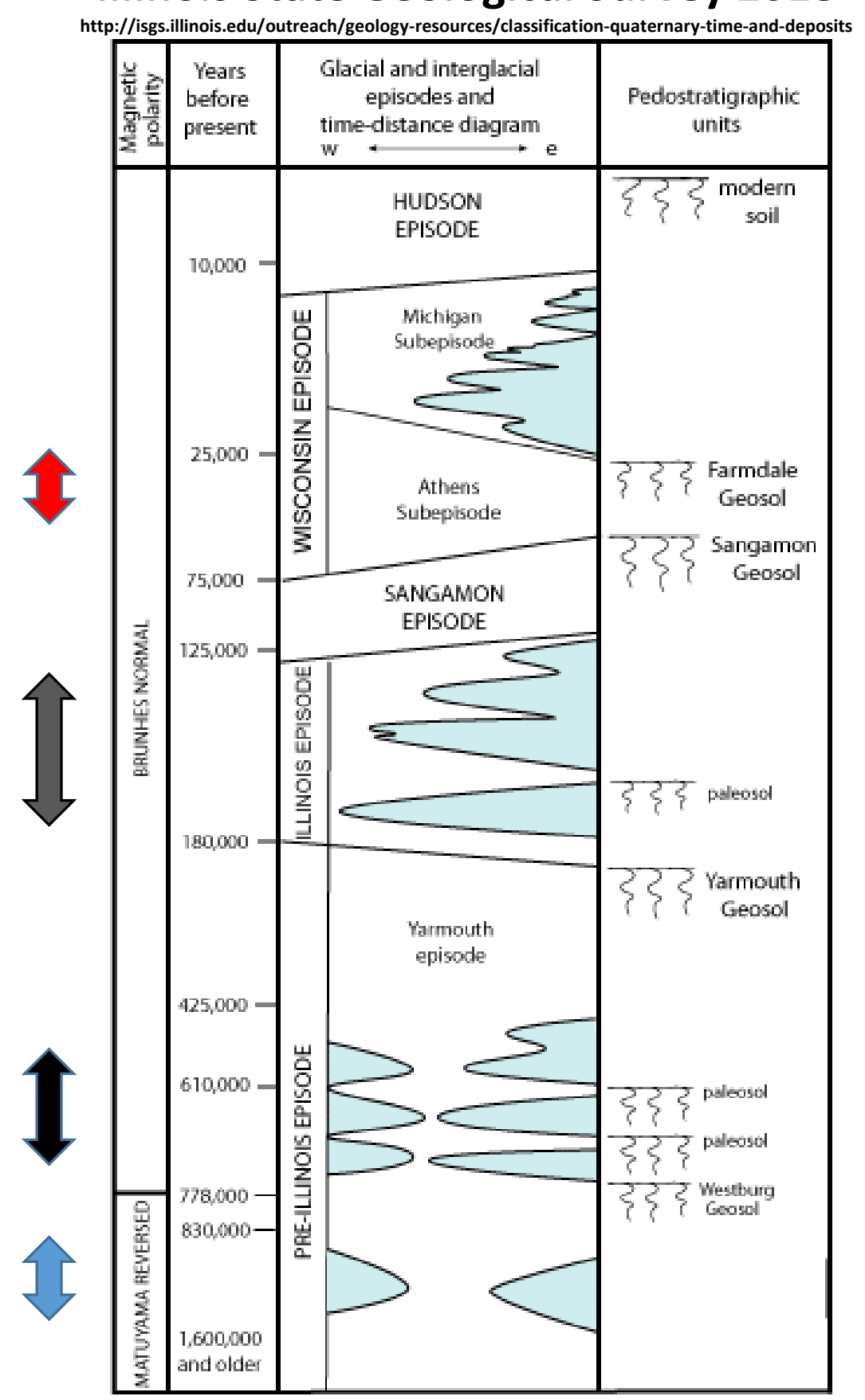




\section{Lake Monongahela Age Assignments}

\begin{tabular}{|c|c|}
\hline Source & Age \\
\hline White 1896 & Pre-Illinoian \\
\hline Campbell 1902 & Pre-Illinoian (Kansan or Pre-Kansan) \\
\hline Leverett 1934 & Pre-Illinoian, Illinoian \& Wisconsin \\
\hline Lessig 1963 & Pre-Illinoian; $1^{\text {st }}$ regional glaciation \\
\hline $\begin{array}{l}\text { Clendening, et al. } 1967 \\
\text { Gillespie \& Clendening } 1968\end{array}$ & $\begin{array}{l}\text { Farmdalian (Wisconsin) } \\
\begin{array}{ll}22,000 \pm 100014 \mathrm{C}-\mathrm{BP} & {[26,394 \pm 1262 \text { CalBP }]} \\
>33,00014 \mathrm{C}-\mathrm{BP} & {[>37,500 \text { CalBP }]}\end{array}\end{array}$ \\
\hline $\begin{array}{l}\text { Jacobson 1985, } 1987 \\
\text { Jacobson et al. } 1988\end{array}$ & $\begin{array}{l}\text { Pre-Illinoian, Illinoian, (Wisconsin Low Terraces) } \\
>40,000 \text { 14C-BP [> 43,700 CalBP] } \\
<780,000 \text { BP (Brunhes) } \\
>780,000 \text { BP (Matuyama) }\end{array}$ \\
\hline $\begin{array}{l}\text { Behling \& Kite } 1988 \\
\text { Kite unpublished data } \\
\text { Morgan } 1994\end{array}$ & $\begin{array}{ll}\text { Pre-Illinoian, Illinoian } & \\
>34,000 \text { 14C-BP } & {[>39,600 \text { CalBP }]} \\
>38,000 \text { 14C-BP } & {[>42,000 \text { CalBP }]}\end{array}$ \\
\hline $\begin{array}{l}\text { Marine 1997; Marine \& } \\
\text { Donahue } 2000\end{array}$ & $\begin{array}{l}\text { Pre-Illinoian \& Illinoian } \\
<780,000 \text { BP (Brunhes) }\end{array}$ \\
\hline
\end{tabular}

Walker \& Lowe, 2007, Journal of the Geological Society 164 (6): 1073-1092

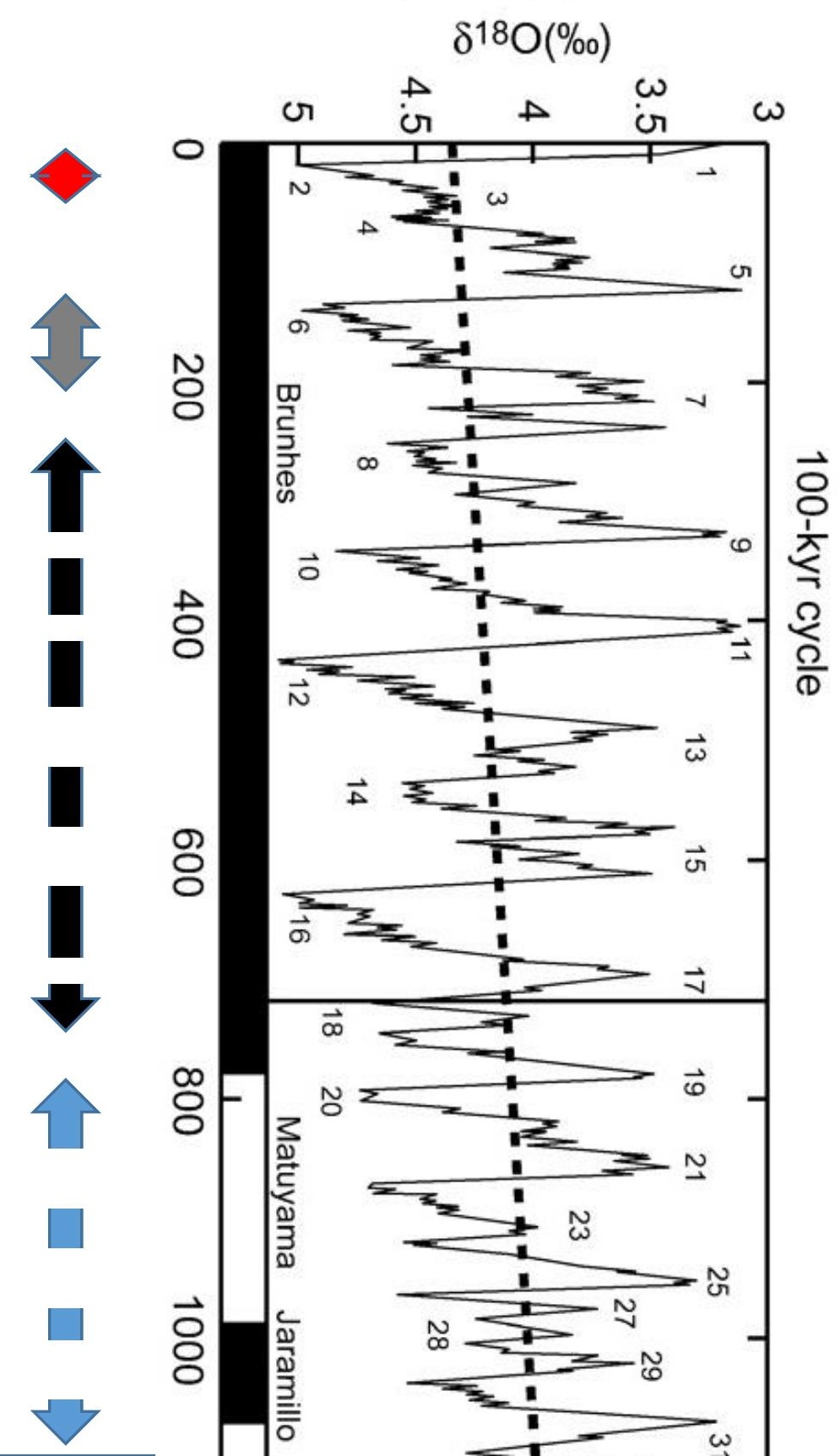




\section{Lake "Mon"}

Reconstruction

(Mark Swift, 2017)

Existing Topography Filled to $335 \mathrm{~m}$ Elevation above Midland, PA

Filled to $330 \mathrm{~m}$ between Midland, PA \& New

Martinsville, WV

How to Reconcile Different Levels on Glacier-Dammed Lake at Traditional Pre-Wisconsin Limit Leverett: Outwash Dammed Lake vs. White's Glacier Dammed Lake
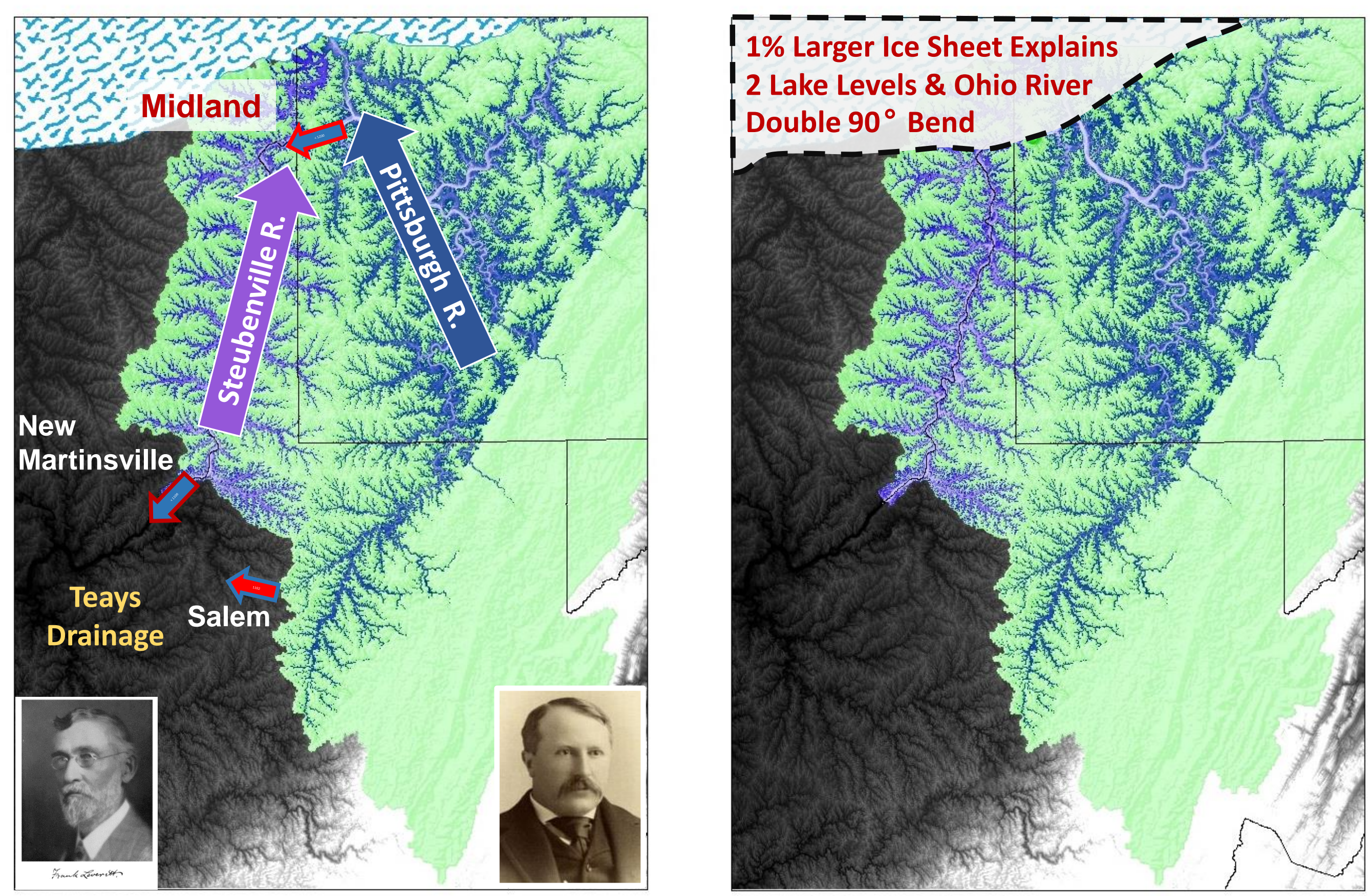


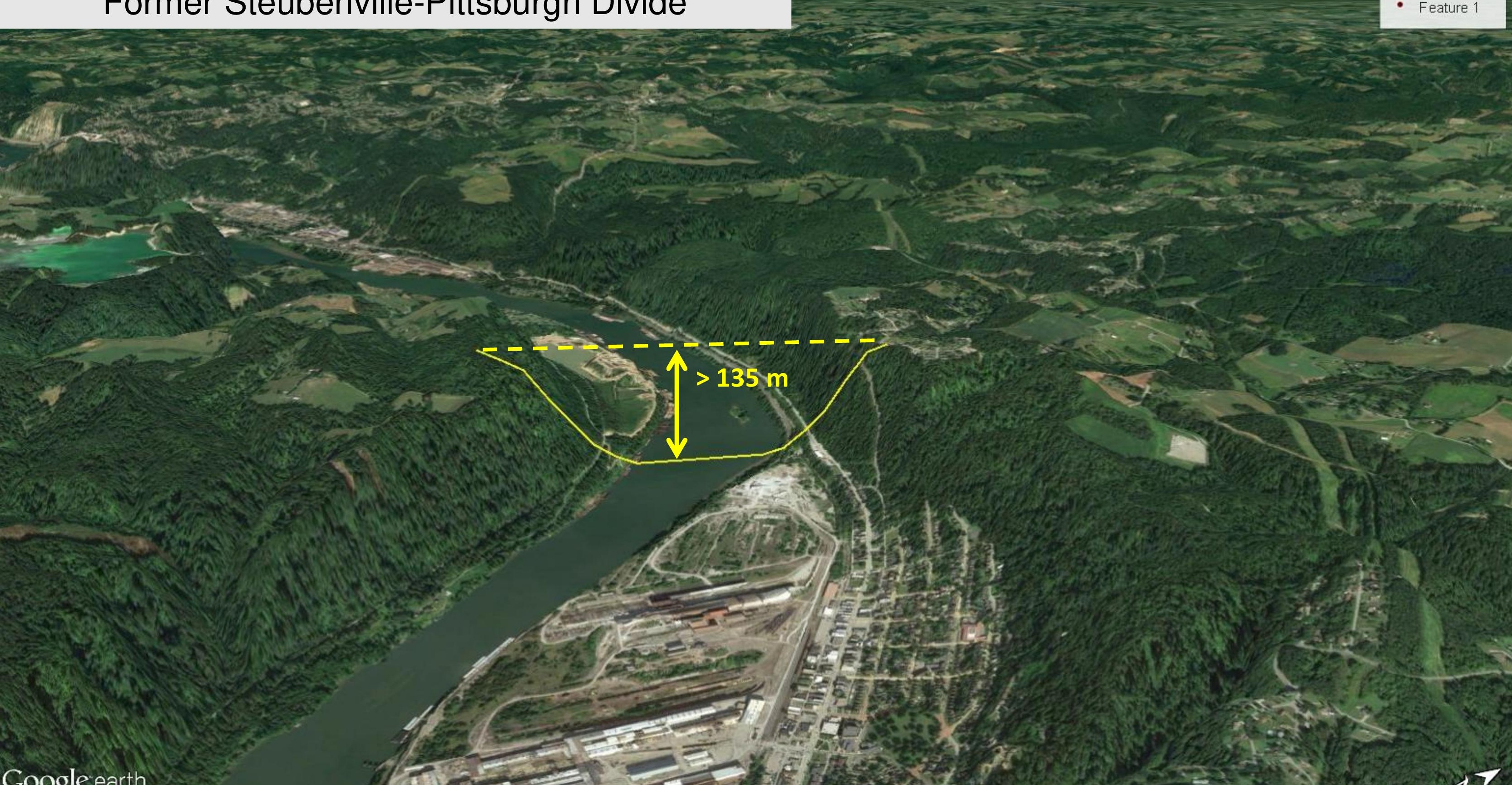




\section{Jacobson (1985)}

Correlations of Upper Ohio River Valley Lacustrine \& Alluvial Terrace Deposits

Ohio River Profiles at Mouths of Outwash Sources (Allegheny, Beaver and Little Beaver Rivers) Suggest $>30 \mathrm{~m}$ ( 100 ft) of Aggradation in Last 3 Glaciations

Quaternary History = Too Complicated to

Reconstruct on Morphology Alone

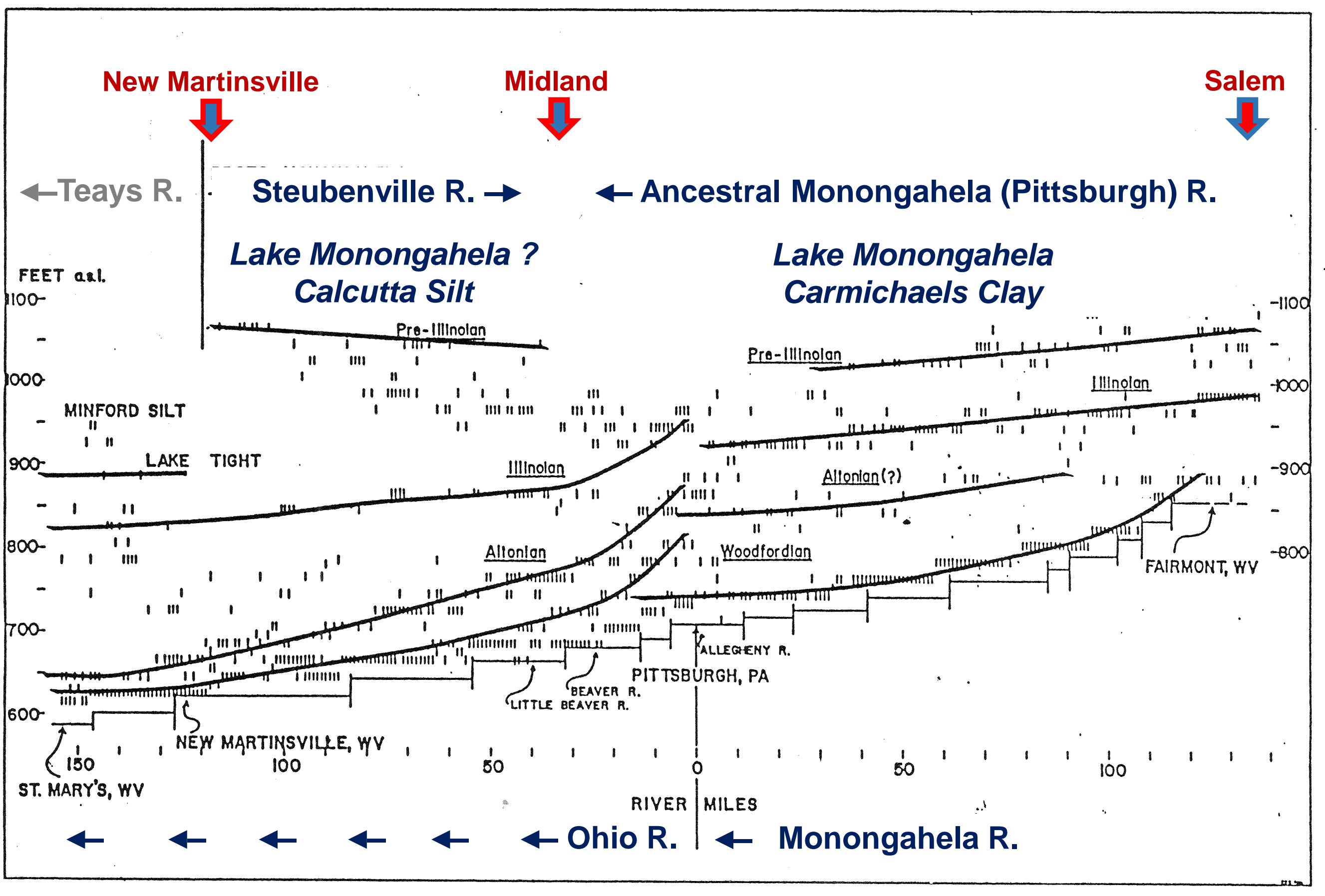




\section{Youghiogheny}

River Terraces

$\sim 3 \mathrm{~km} \mathrm{NW}$ of Connellsville, PA

W.O. Hikock Photo, July 1934

Published in Pennsylvania Geology Fall 2018, p. 21

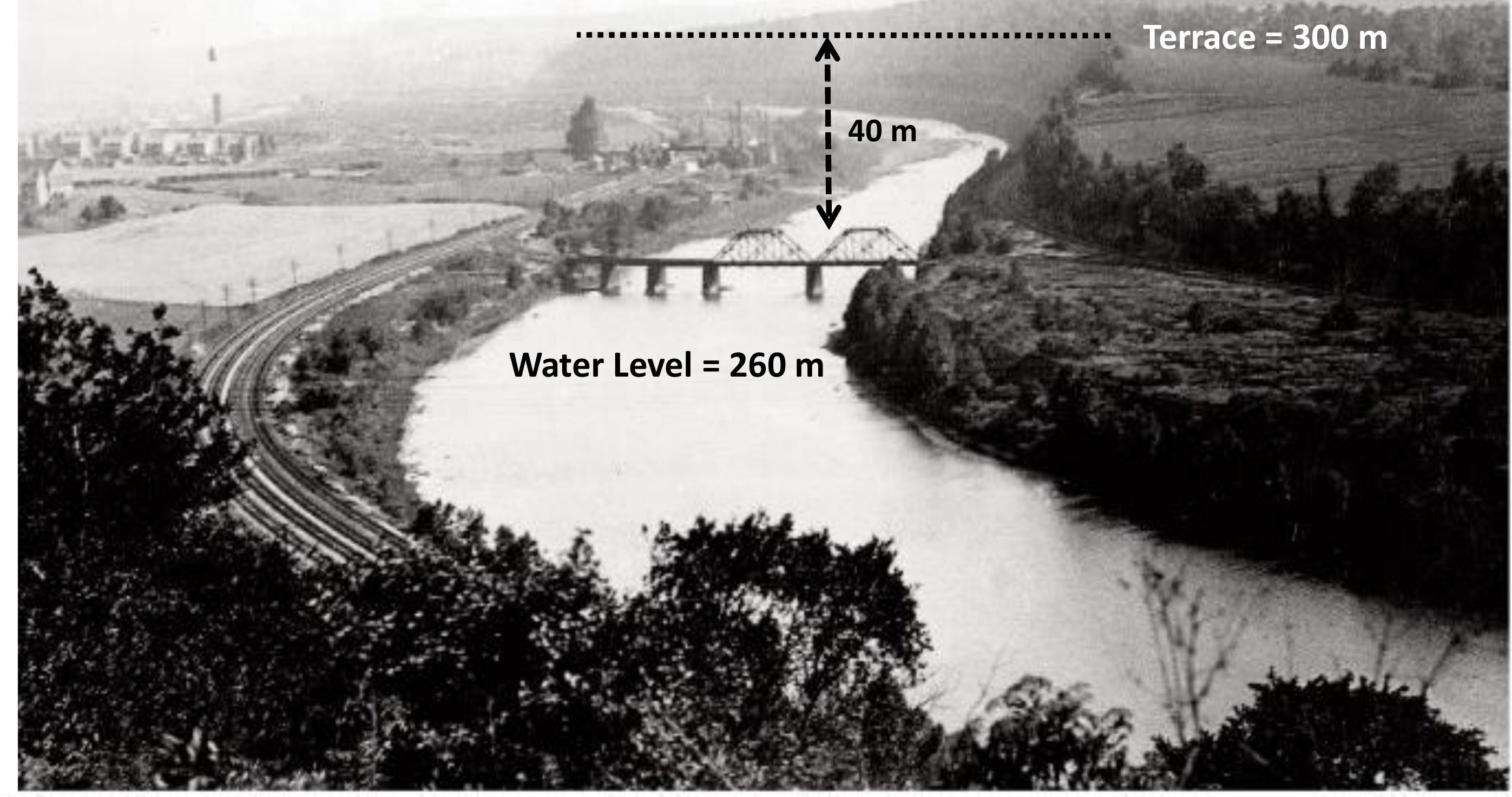




\section{WVU Woodburn Circle Excavation June 2017}

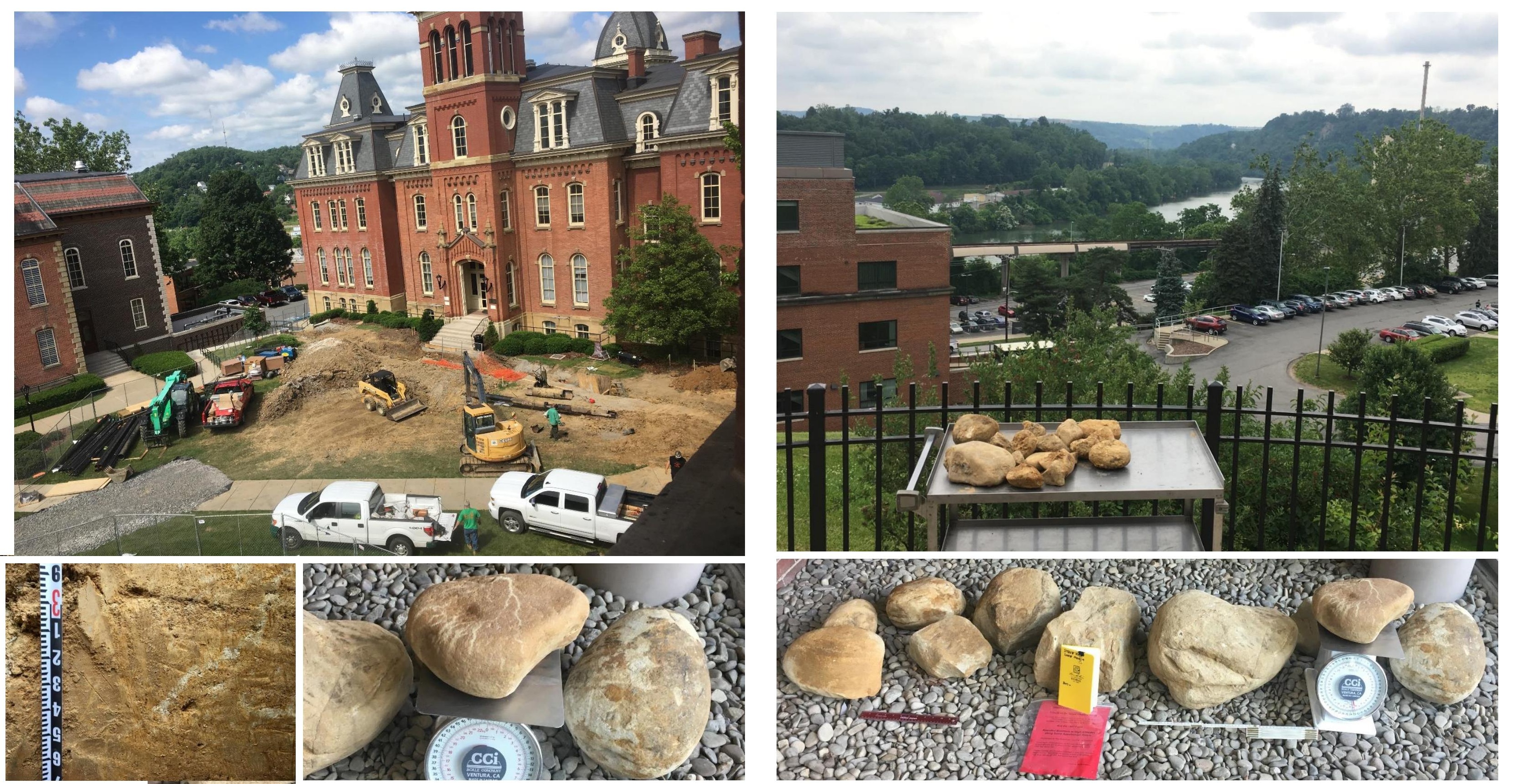




\section{WVU Campus \& Old Mountaineer Field $\sim 1924$}

Monongahela: Unami (Algonquian) Word Meaning "Falling Banks"

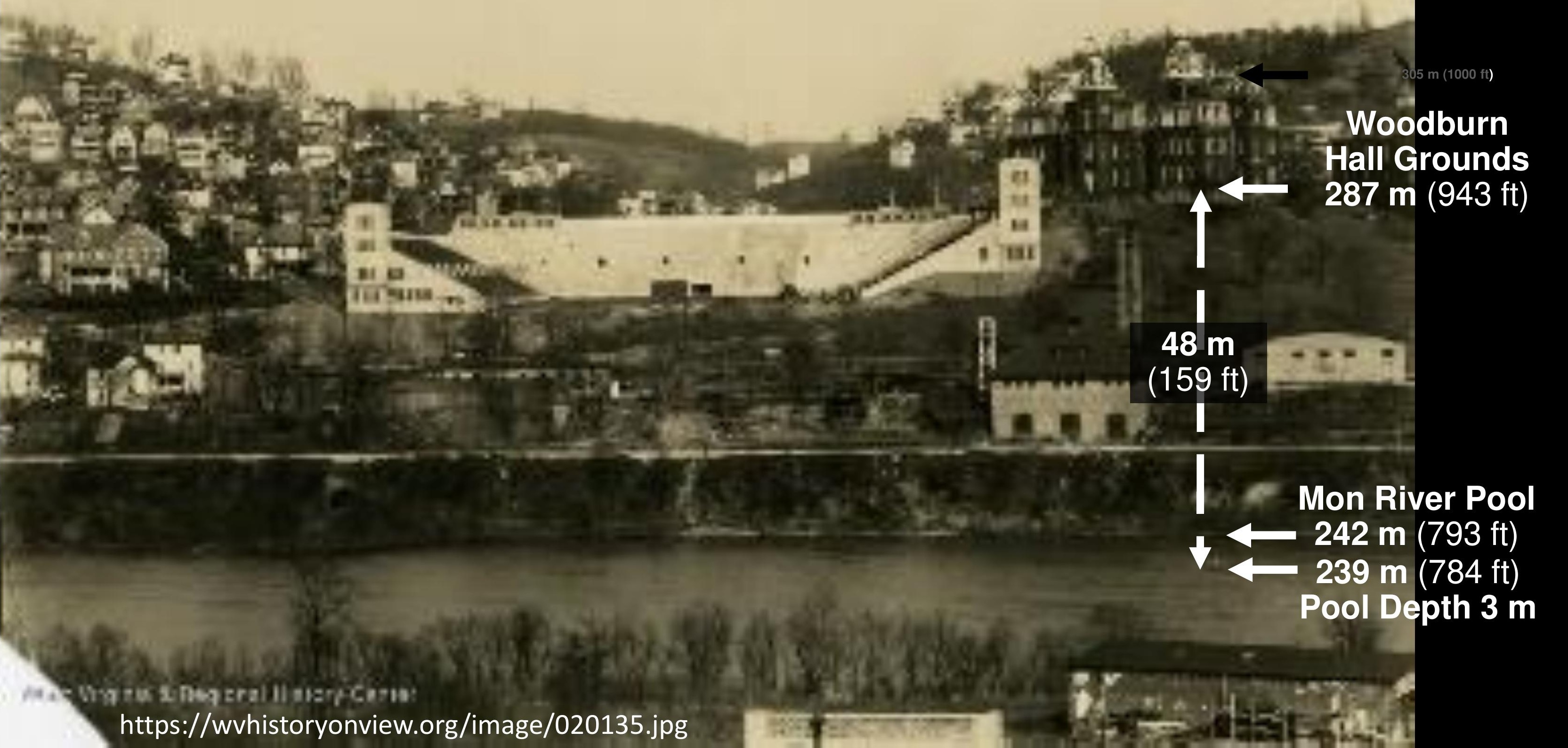




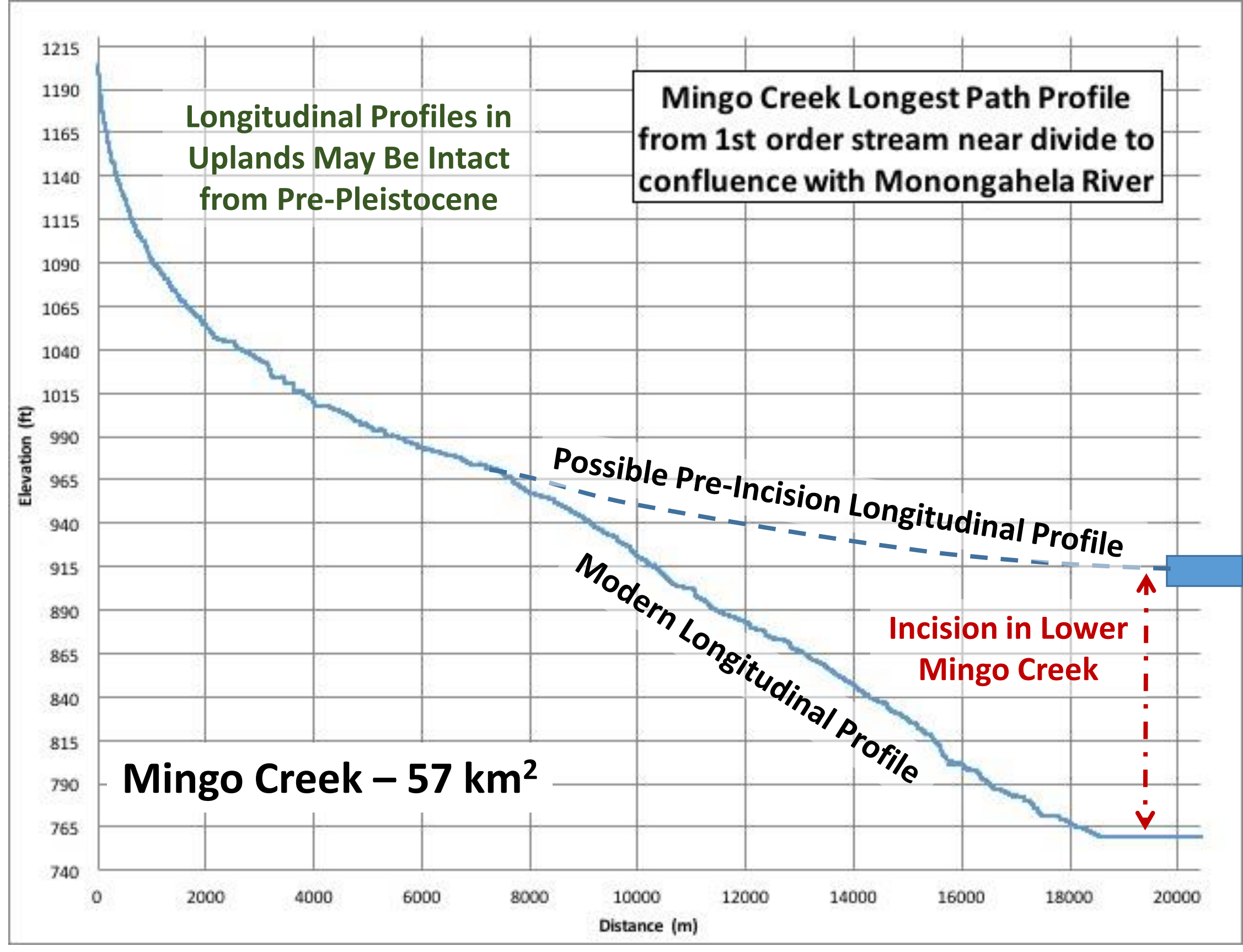

\title{
Effects of River Incision on Tributaries
}

\author{
Mark Swift, \\ WVU Geography \\ M.A. Research
}

Ancestral Monongahela River (Pittsburgh River) Terrace $\sim 900 \mathrm{ft}$ 
Mingo Creek- Zones With Downstream Gradient Steeper than Upstream Gradient

Measurement Distance $(\mathrm{d})=16 \mathrm{~km}, 14 \mathrm{~km}, 12 \mathrm{~km}, 10 \mathrm{~km}, 8 \mathrm{~km}, 6 \mathrm{~km}$

Lower Reach Gradient $>0.001$ and Ratio of Lower Reach to Upper Reach $>1.2$

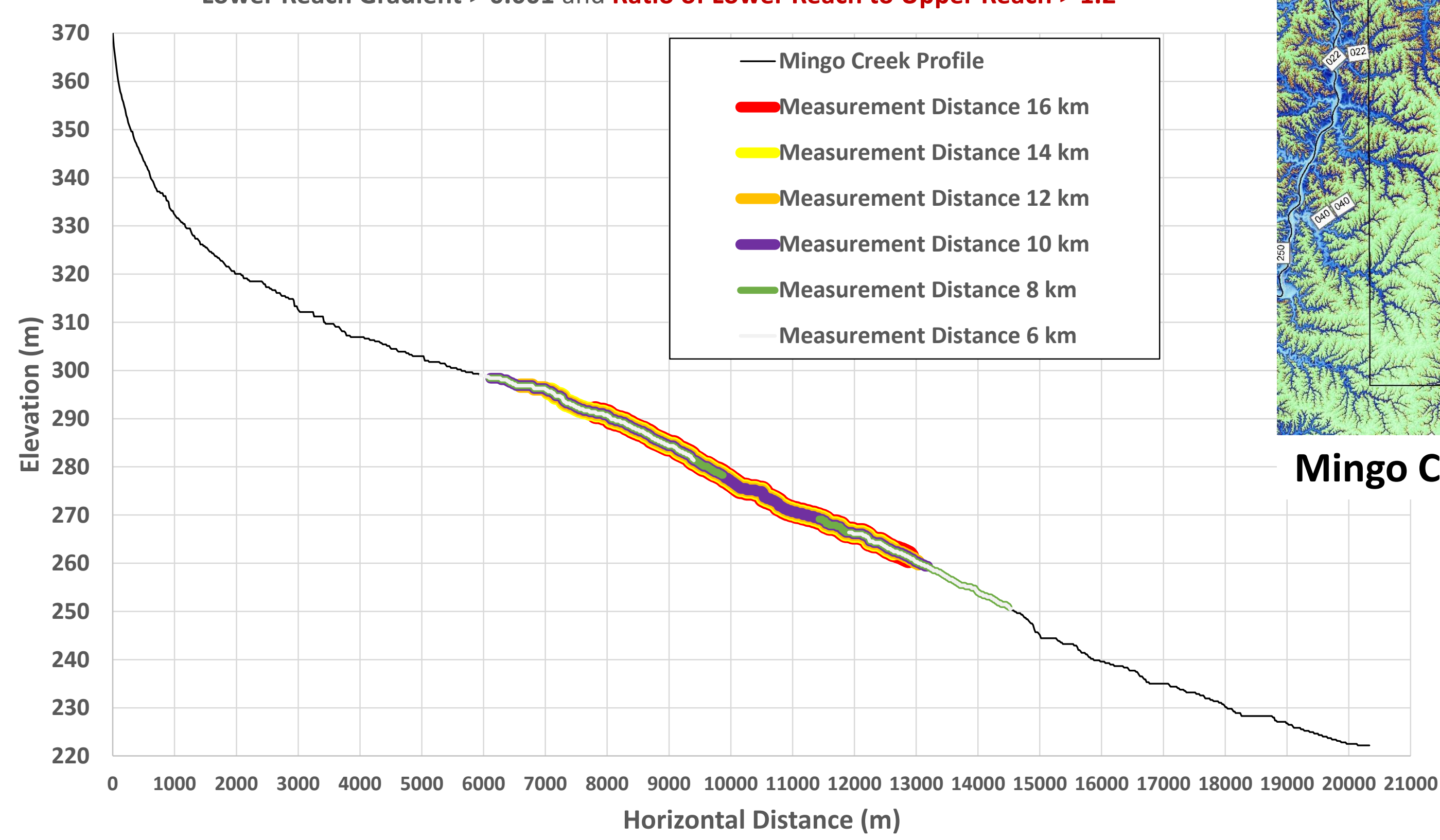


Muddy Creek - Zones With Downstream Gradient Steeper than Upstream Gradient

Measurement Distance (d)= 32 km, 28 km, 24 km, 20 km

Lower Reach Gradient > .001 and Ratio of Lower Reach to Upper Reach > 1.2

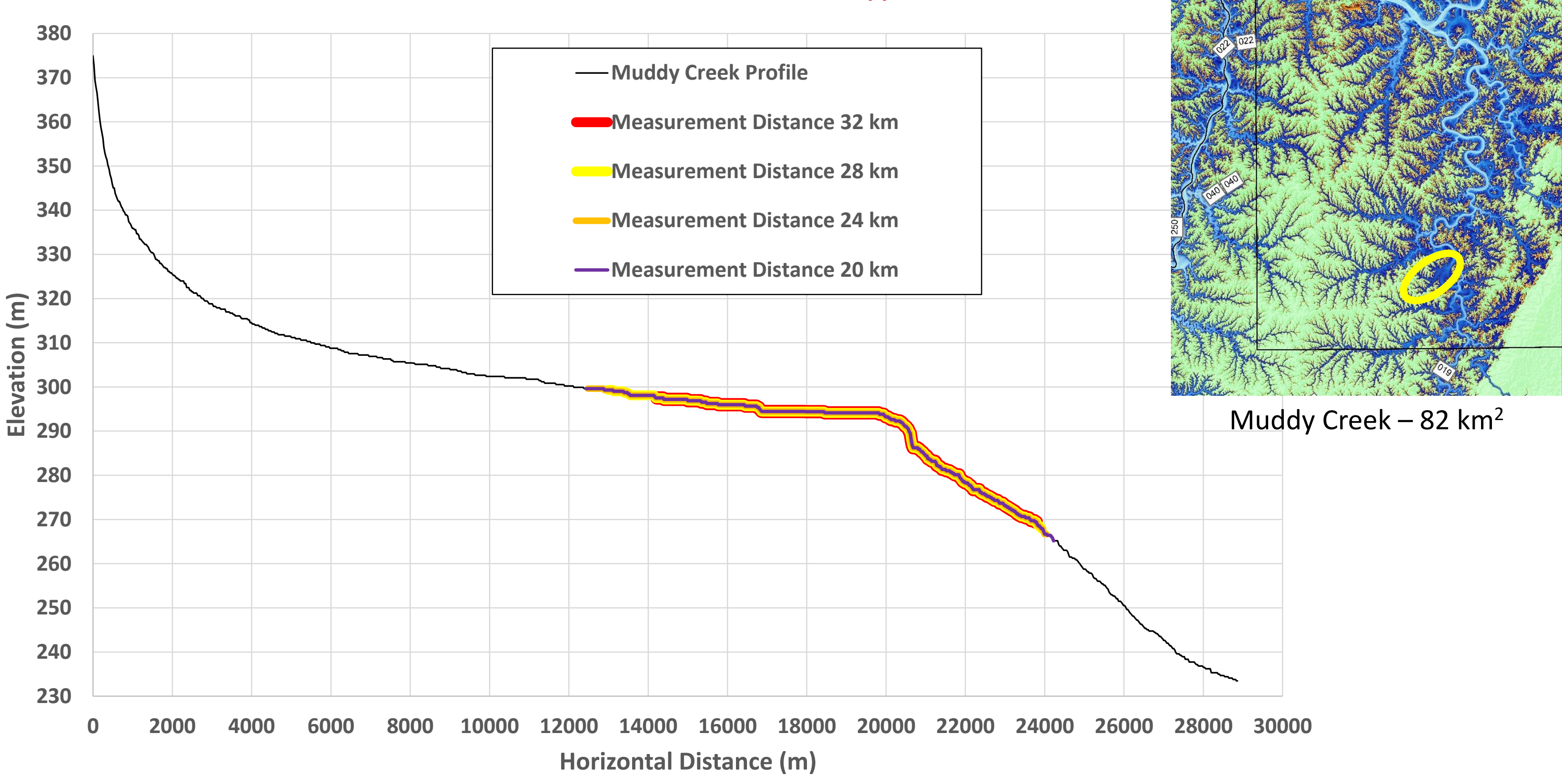


Little Whiteley Creek - Zones With Downstream Gradient Steeper than Upstream Gradient Measurement Distance $(\mathrm{d})=12 \mathrm{~km}, 10 \mathrm{~km}, 8 \mathrm{~km}, 6 \mathrm{~km}$

Lower Reach Gradient > .001 and Ratio of Lower Limb to Upper Limb > 1.2

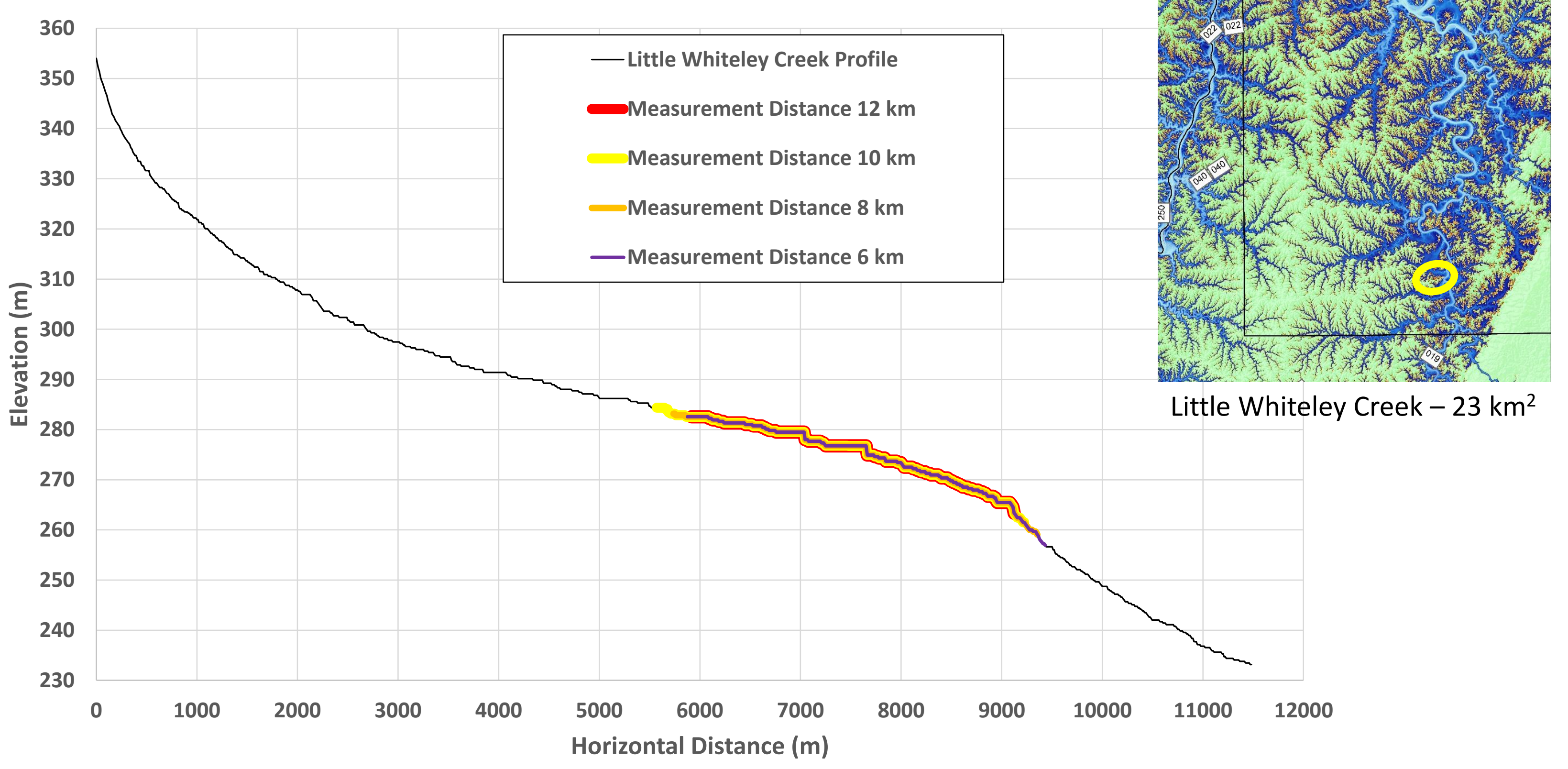




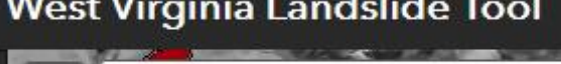

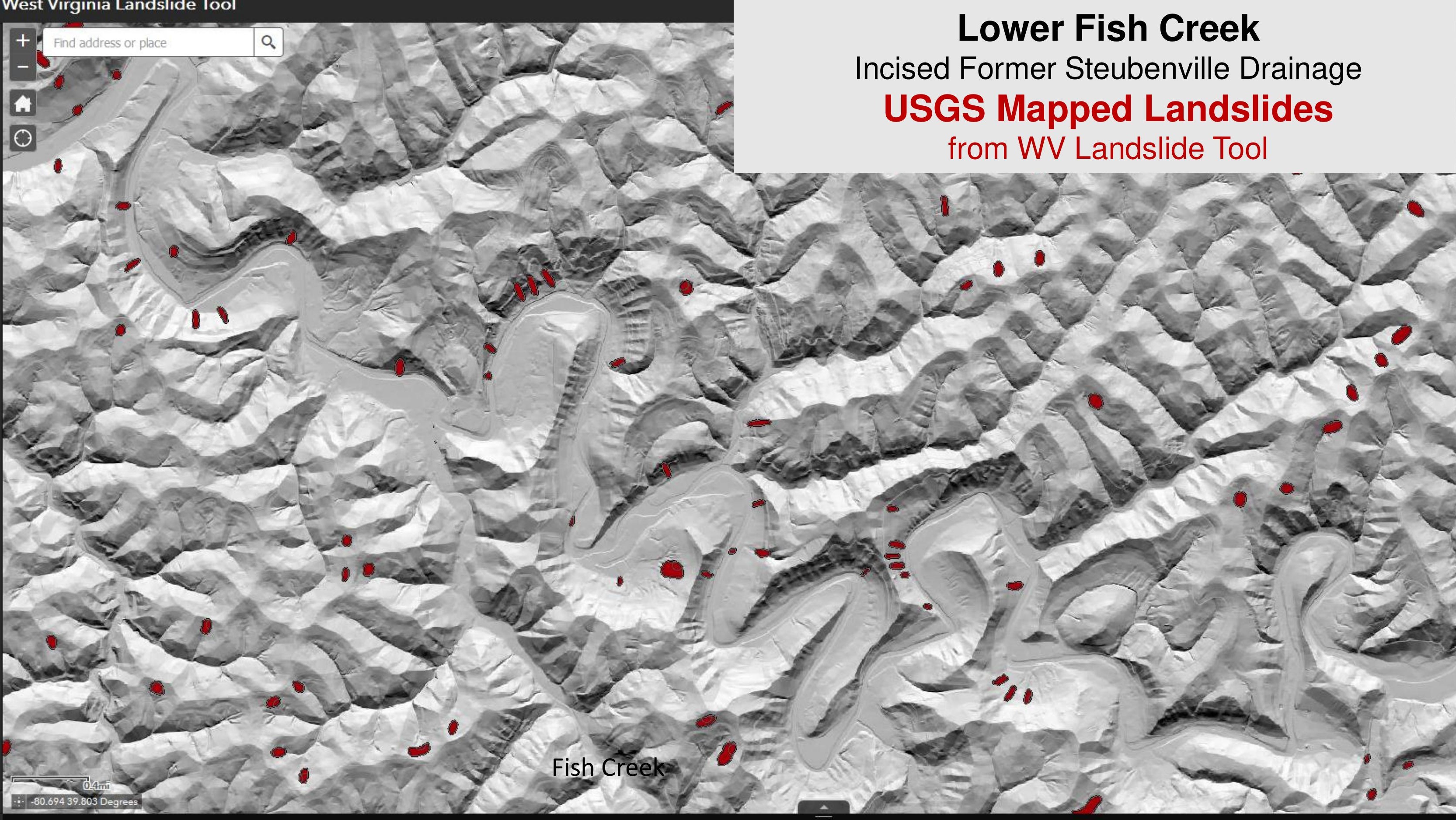


- Much of Upper Ohio River Basin Has "High Landslide Incidence"

- West Virginia $=1 / 9^{\text {th }}$ of $1973-1983$ Landslide Damage in 48 States \#1 in Per Capita Landslide Damage (Brabb, 1984, USGS OF 84-486).

Coterminous U.S. Landslide Overview Map USGS PP 1183

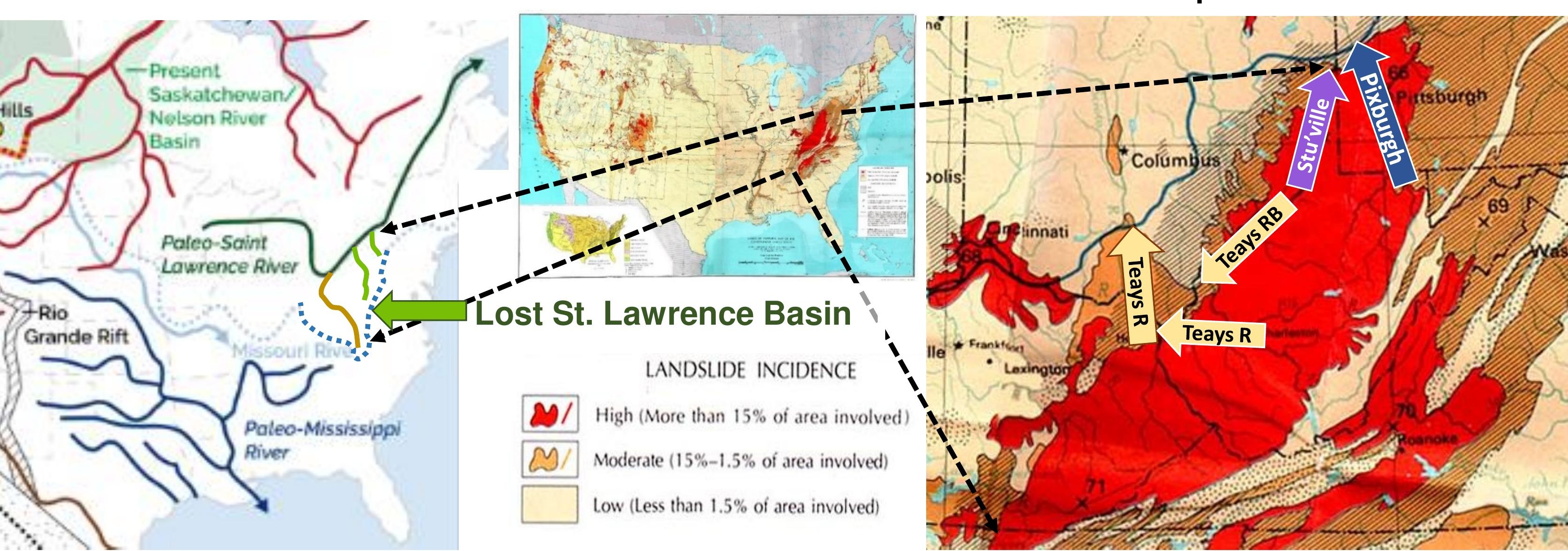

